\title{
An articulated handle to improve the ergonomic performance of robotic dextrous instruments for laparoscopic surgery
}

\author{
B. Herman ${ }^{1,2}$, A. Devreker ${ }^{1, *}$, F. Richer ${ }^{3}$, A. Hassan Zahraee ${ }^{3}$, and J. Szewczyk ${ }^{3}$ \\ ${ }^{1}$ Université catholique de Louvain, Institute of Mechanics, Materials, and Civil Engineering, \\ Center for Research in Energy and Mechatronics, Louvain-la-Neuve, Belgium \\ ${ }^{2}$ Université catholique de Louvain, Louvain Bionics, Louvain-la-Neuve, Belgium \\ ${ }^{3}$ Université Pierre et Marie Curie - CNRS, Institute for Intelligent Systems and Robotics, Paris, France \\ *now with: K. U. Leuven, Department of Mechanical Engineering, Division Production engineering, \\ Machine design and Automation, Leuven, Belgium
}

Correspondence to: B. Herman (benoit.herman@uclouvain.be)

Received: 19 November 2013 - Revised: 2 April 2014 - Accepted: 8 May 2014 - Published: 23 May 2014

\begin{abstract}
Hand-held robotic instruments with dextrous end-effectors offer increased accessibility and gesture precision in minimally invasive laparoscopic surgery. They combine advantages of both intuitive but large, complex, and expensive telesurgery systems, and much cheaper but less user-friendly steerable mechanical instruments. However, the ergonomics of such instruments still needs to be improved in order to decrease surgeon discomfort. Based on the results of former experimental studies, a handle connected to the instrument shaft through a lockable ball joint was designed. An experimental assessment of ergonomic and gesture performance was performed on a custom-made virtual reality simulator. Results show that this solution improves ergonomics, demanding less wrist flexion and deviation and elbow elevation, while providing gesture performance similar to a robotic dextrous instrument with standard pistol-like handle configuration.
\end{abstract}

\section{Introduction}

Several gestures in laparoscopic surgery are rather difficult to perform with conventional non-dextrous instruments. Such a straight and elongated instrument passing through a cannula has only a reduced set of 4 degrees of freedom (DOFs). As a consequence, the orientation of the instrument end-effector is coupled to its position in the abdominal cavity, and by extension to the handle posture above the patient. These kinematic constraints often force surgeons to work in awkward and painful postures, inducing discomfort and even pain after a while (Nguyen et al., 2001).

Adding one or several distal DOFs can help to restore mobility as in open surgery, making complex gestures like suturing easier. The da Vinci surgical system (Intuitive Surgical Inc., Sunnyvale, CA) offers such a functionality, and the benefits in terms of ease of use and ergonomics are well established (Freschi et al., 2013). However, its wide diffusion and use in clinical routine are restricted by the high selling price and maintenance costs of this telesurgery robotic system, and by its size and bulk.

A couple of simpler hand-held dextrous instruments have been available since 2006, such as RealHand HD from Novare Surgical Systems, Cupertino, CA (Danitz, 2006), Radius from Tuebingen Scientific Medical GmbH, Tübingen, Germany (Schwarz et al., 2005), Roticulator from Covidien Inc., Mansfield, MA (Marczyk et al., 2013), or Autonomy LaparoAngle from Cambridge Endo, Framingham, MA (Lee and Chamorro, 2008). Research prototypes are also under development (e.g. Awtar et al., 2012; Wang et al., 2012). The distal DOFs of these devices are actuated manually either via knobs or joysticks on the handle (fingertip control), or through a jointed handle that is mechanically coupled to the end-effector (wrist control). They are very useful especially for single-port laparoscopic procedures (MacDonald et al., 2009), where the two instruments are inserted into the same 
cannula and cross each other in the abdominal wall, requiring intra-abdominal bending to reach the same site in a triangulated configuration (Rettenmaier et al., 2009; Frede et al., 2007; Rosenblatt et al., 2012; Endo et al., 2011). Kolwadkar et al. (2011) demonstrated experimentally that a fingertipcontrolled instrument with a miniature joystick outperforms a wrist-controlled instrument on a needle driving task in conventional laparoscopy. The same conclusion was reached by Okken et al. (2012). However, the latter study also showed that, in some cases, conventional straight instruments performed even better than both dextrous prototypes. According to the authors, this can be due to the difference in quality between commercialized standard instruments and their research prototypes, and to the absence of locking mechanisms for the jointed handle and the joystick. However, adding locking mechanisms to a miniature joystick might not be that straightforward, especially if one wishes to lock one DOF and leave the other free. For example, to perform a stitch in a plane that is not orthogonal to the longitudinal axis of the instrument, the optimal strategy is to actuate the terminal roll motion of the grasper under a constant yaw bending (Hassan Zahraee et al., 2010). Furthermore, a direct mechanical transmission that synchronizes joystick and end-effector angular configurations (position-position mapping) requires a smooth and continuous finger motion to actuate the intraabdominal DOFs, and restricts their range of motion.

One solution to these limitations is to replace the direct mechanical link by actuators whose velocity can be controlled by the joystick (position-velocity mapping). This has several advantages over a mechanical transmission. Distal range of motion is not restricted by the joystick angular range. Using non-backdrivable transmissions between actuators and end-effector induces a self-locking behavior: if the surgeon does not tilt the joystick, the intra-abdominal DOFs remain at rest. In addition, while a high amount of force must be applied by the finger on the joystick to stitch with a mechanical transmission, the required force and torque are now provided by the actuators. Finger and hand muscular fatigue can therefore be reduced. We demonstrated on a custom-made virtual-reality (VR) simulator that the number of successful stitches was significantly higher when controlling the distal DOFs with a thumb-actuated joystick mounted on the instrument handle in a position-velocity mapping, with respect to a jointed handle with position-position mapping and a locking feature (Hassan Zahraee et al., 2010). Hand-held robotic instruments might thus combine the advantages of both solutions: a cost-effective dexterity as in mechanical dextrous instruments; with a user-friendly control of distal DOFs as offered by telesurgery systems. Three hand-held robotic instruments have so far been put on the market, all using the optimal yaw-roll kinematics: JAiMY from EndoControl Medical, La Tronche, France (Paik et al., 2010), Kymerax from Terumo Europe Advanced Surgical, Eschborn, Germany (Hackethal et al., 2012) and Dextérité Hand Held Robot from Dextérité Surgical, Annecy, France
(Barrier et al., 2010). Various research prototypes were also introduced by the University of Tokyo, Japan (Yamashita et al., 2004), Toshiba Medical Systems with the Keio University School of Medicine, Tokyo, Japan (Jinno et al., 2002), the University of Darmstadt, Germany (Röse et al., 2009), Scuola Supereriore Sant'Anna, Pisa, Italy (Piccigallo et al., 2008), and the Delft University of Technology (Lassooij et al., 2012). These instruments help surgeons with their biggest challenge which is performing complex gestures in laparoscopy. However, they fail to completely address another very important problem of laparoscopists - the poor ergonomics of laparoscopic surgery.

Decoupling handle and shaft orientation with a free ball joint between them allows exploration of the entire intraabdominal workspace without excessive wrist flexion or deviation, since the handle is not forced to rotate while swiveling the shaft. We showed on our VR simulator (Herman et al., 2011) that the ergonomic performance of such an articulated handle was significantly higher, but at the cost of a lower gesture performance (i.e. an increase in task duration and path length). This can be attributed mainly to the "floating" behavior of the free handle that might complicate reaching a precise target by inducing some loss of shaft controllability. To solve this problem of floating behavior, introducing the possibility to lock/unlock the joint between handle and shaft seems to be a promising approach. The Dextérité Hand Held Robot also features a lockable joint between the handle and the shaft. However, it is only a 1-DOF revolute joint that allows a Yaw motion, which might not be sufficient for releasing all strains in the surgeon's wrist. In this paper, we introduce the design and performance assessment of a robotic instrument equipped with two lockable joints between handle and shaft that provide 3 revolute DOFs. Our hypothesis is that it could allow a more comfortable arm and wrist configuration regardless of the shaft orientation, while restoring instrument rigidity for a precise control of fine surgical gestures. The remainder of this paper is organized as follows: Sect. 2 describes the handle mock-up and its locking modes. An experimental performance assessment using the VR simulator is detailed in Sect. 3. Results are reported in Sect. 4 and discussed in Sect. 5.

\section{Articulated handle}

\subsection{Design overview}

Figure 1 depicts the prototype of a lockable articulated handle. It is composed of a Nunchuk handle (Nintendo) with a thumb-actuated 2-DOF joystick that allows the control of the Yaw and Roll distal DOFs. Note that, for this study, these distal DOFs are implemented virtually in the simulator, whereas the actual robotized prototype (not described here) embeds DC motors to actuate them. The Nunchuk handle is connected to the instrument shaft through two lockable joints: 


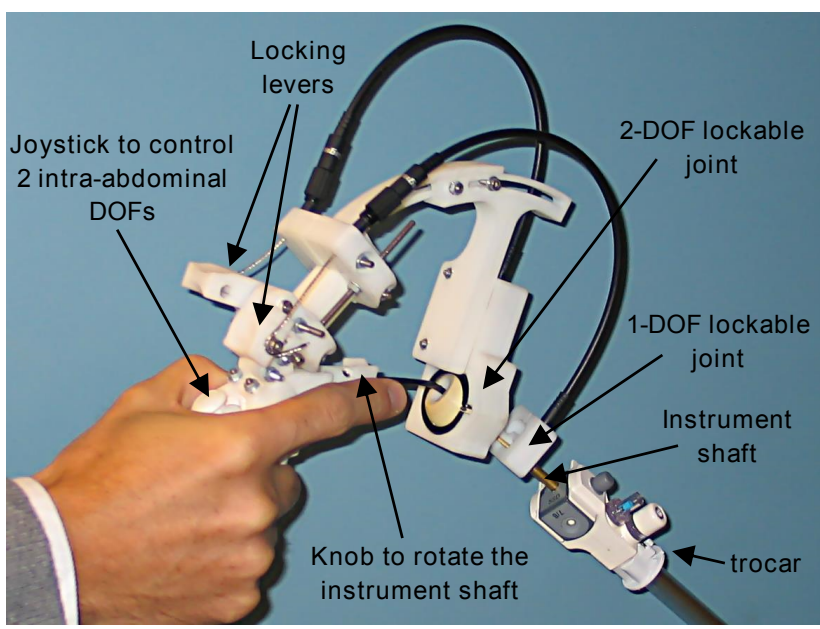

Figure 1. Prototype of articulated handle with lockable joints.

a 2-DOF universal joint and a 1-DOF revolute joint around the shaft longitudinal axis.

As shown in Fig. 2, the universal joint is made up of a plastic ball joint with a pin partly inserted into the ball, perpendicularly to the instrument shaft. The pin can slide along a straight groove in the joint base to allow Yaw and Pitch motions of the shaft, but prevents any Roll motion (i.e. selfrotation). The universal joint allows the user to move the shaft within a cone of approximately $70^{\circ}$ of aperture, while self-rotation is unrestricted. A star-shaped knob at the front of the handle is linked to the instrument shaft via a flexible axle in order to control its Roll motion with the index finger, as on most straight instruments.

Both joints are locked by means of a compression spring and can be released by a thumb-actuated lever that pulls a Bowden cable. The locking torque produced by the spring on the universal joint is $0.5 \mathrm{Nm}$ for Pitch (i.e. left-right motion of the instrument tip) and $0.25 \mathrm{Nm}$ for Yaw (i.e. up-down motion). It is obviously not sufficient to perform a task in the real surgical world, where a force of up to $50 \mathrm{~N}$ may be required to insert a surgical needle into muscle tissue. Nevertheless, since it is designed to operate in a VR simulator without any interaction force between the instrument tip and the environment, the only forces that the locking mechanism has to withstand are the reaction and friction forces in the trocar (neglecting the shaft's own weight). Furthermore, we determined experimentally that the locking torque is one order of magnitude higher than the friction torque inside the joint. The user can thus easily feel if the joint is locked or free during a trial run on the VR simulator, which is the purpose of this mock-up.

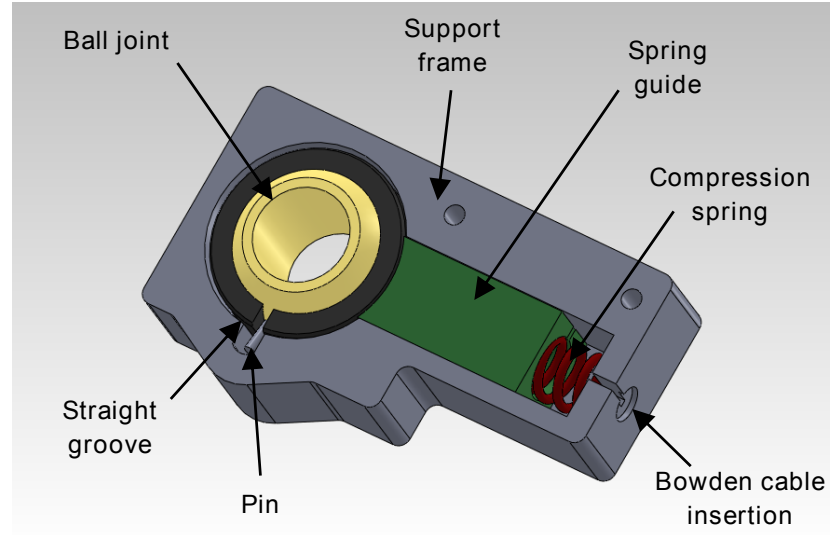

Figure 2. CAD view of the universal joint with locking compression spring.

\subsection{Locking modes}

The handle can be used in one of these four modes:

1. Standard fixed handle, with both joints locked in their central position.

2. Fully free handle, with both joints released during the entire task, the shaft self-rotation being controlled by the index finger.

3. 2-DOF adjusted and locked, 1-DOF free, in which the 2-DOF joint is locked in a convenient configuration during the entire task while the 1-DOF joint can be actuated by finger during the task.

4. Both joints adjusted and locked in a convenient configuration during the entire task.

The first two modes are similar to those tested previously in Herman et al. (2011), although the actuated self-rotation of the shaft through the active trocar was replaced by finger actuation using the star-shaped knob described above. For the last two modes, the handle is pre-locked in a convenient and ergonomic configuration after positioning the end-effector in the task region, before starting the task itself. According to Matern and Waller (1999), this configuration is ideal with the arm slightly abducted, retroverted, and rotated inwards at shoulder level, the elbow bent at about 90 $120^{\circ}$, and the hand in medial configuration (i.e. without any pronation, wrist flexion or deviation). The pre-locking procedure is compatible with actual surgery, during which instrument motions remain confined in a small region of the intraabdominal workspace for a few minutes to perform a specific action (e.g. dissection, cutting, clip placement, stitching). 


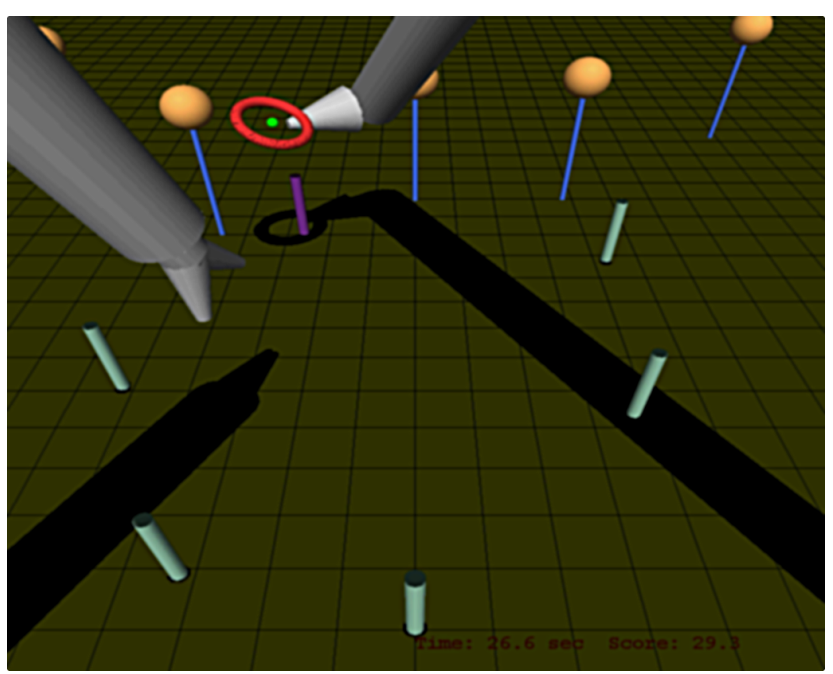

Figure 3. Screen capture of the VR simulator pick-and-place task.

\section{Performance assessment}

The performance of these four modes was assessed through an experiment on the aforementioned VR simulator. This section describes the manipulation task, the two main comparison metrics, the experimental setup and protocol, and the statistical methods used to analyze recorded data. Since the present study derives directly from the experiment reported in Herman et al. (2011), only important information and differences with the previous study are summarized in this paper. The reader interested in further details will find them in the aforementioned reference.

\subsection{Task}

A single pick-and-place task (Fig. 3) was used to assess the performance of the four handle modes. It reproduces the gesture complexity of stitching but, contrary to the latter, remains feasible with the same degree of difficulty in the absence of haptic feedback. As explained above, the task was improved compared to the previous experiment so as to reduce the instrument motion required during a task, by placing the ring close to the pin at the beginning of the trial. In addition, the pin can be placed either vertically (normal to the bottom plane) or at $45^{\circ}$ from the vertical, so as to require a ring orientation during the task.

The chronometer is triggered when the subject bursts a balloon placed on top of a pin with the instrument tip. He/she must then grasp a ring placed horizontally on the bottom of the workspace, next to the pin. The grasp must be performed on a specific portion of the ring and under a certain orientation by using the intra-abdominal DOFs. This mimics the fact that a curved needle must be grasped under specific conditions to perform a stitch. Finally, the ring must be

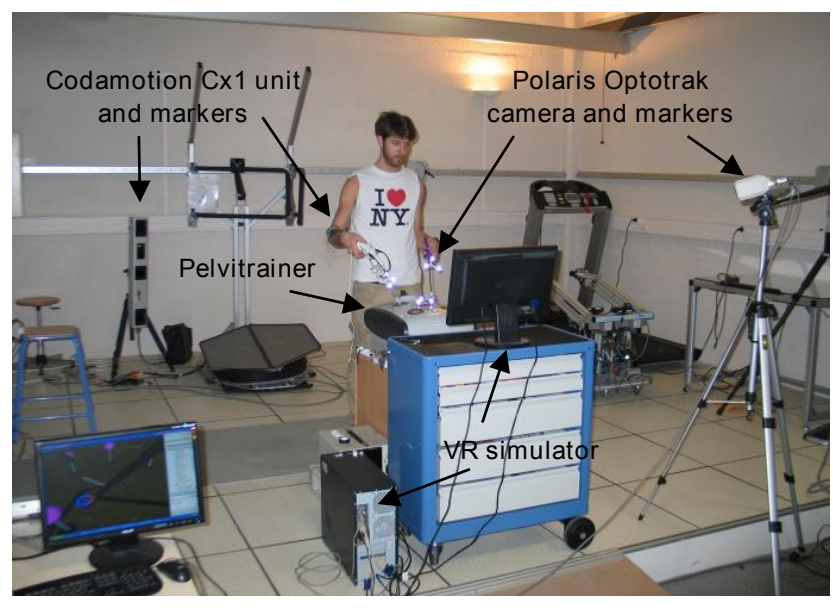

Figure 4. Overview of the experimental setup.

placed on the pin after a reorientation of the end effector. The chronometer is stopped when the ring is released on the pin.

\subsection{Metrics}

The performance of the four handle modes was assessed using the same metrics as in Herman et al. (2011): a global gesture performance score available in most commerciallyavailable laparoscopy simulators, and an instantaneous ergonomic score.

The global performance score $(P)$, initially proposed by Huang et al. (2005), combines the time to complete the task (TTC), the number of errors (Err) and the motion economy (ME), defined as the ratio between actual and optimal (shortest) path length:

$P=$ TTC + Err $+2 \times$ ME.

The weight for motion economy had been chosen previously in agreement with surgeons for the study reported in Herman et al. (2011).

The ergonomic score is based on a real-time index inspired by the Rapid Upper Limb Assessment (RULA) form (McAtamney and Corlett, 1993) that was adapted by Person et al. (2001) to laparoscopic surgery. This instantaneous score is computed from joint angles of the major upper arm (i.e. the one that holds the dextrous instrument). It ranges over an integer scale from 1 (excellent posture) to 9 (very poor ergonomics). All fine details regarding the computation of this instantaneous score are reported and depicted in Herman et al. (2011).

\subsection{Experimental setup}

The experimental setup is shown in Fig. 4. The mock-up of robotic instrument with an articulated handle and one standard laparoscopic grasper (not required to perform the task but used to make the situation more realistic) are inserted in a 
pelvitrainer through trocars that form a $10 \mathrm{~cm}$ equilateral triangle with a virtual laparoscope. The pelvitrainer and monitor heights are adjusted to respect the standard ergonomic prescriptions for laparoscopy. Positions and orientations of both instruments with respect to the pelvitrainer are measured using a Polaris Optotrak system (Northern Digital Inc., Waterloo, Canada) connected to the VR simulator. To compute the instantaneous ergonomic score described above, major upper limb segments are tracked using a Codamotion system (Charnwood Dynamics Ltd., Leicestershire, UK) consisting of a Hub computer unit and $3 \mathrm{Cx} 1$ units, offering a tracking accuracy better than $0.3 \mathrm{~mm}$.

\subsection{Protocol}

Six right-handed subjects with no experience in surgery and variable experience in playing music or video games took part in the experimental campaign. At the beginning of each session with a new participant, the basic principles of laparoscopic surgery were explained. Then, the purpose of the study was detailed, along with the two metrics. The experimental setup and protocol were briefly explained during the placement of the Codamotion tracking markers on the subject. After calibration of the markers, the VR simulator and the handle were introduced via a short demonstration. The participant was then asked to perform the tasks as quickly as possible and using the shortest possible path, avoiding errors (i.e. a collision between ring and pin, or an instrument out of sight). This instruction was repeated regularly during the session.

Before starting the experiment, a learning exercise similar to the task was performed so as to become familiar with the VR simulator and the instrument. The learning curve for the global performance score and the task duration were fitted after each learning trial using Matlab. The exercise was repeated until the subject reached a performance plateau. On average, 10 repetitions were required. The learning exercise was also repeated after each handle mode change before starting the 5 recorded trials, until the subject felt comfortable with the new mode and the global performance score became stable. Three to five repetitions of the learning exercise were required, depending on the subject and the handle mode.

The task was repeated five times with each handle mode, each repetition being placed at one specific position in the virtual abdomen and under a specific angle so as to cover the entire workspace. The same order of pin placement and orientation was repeated for all handle modes and participants. The experiment lasted $75 \mathrm{~min}$ on average, including initial introduction, learning and experimental phases, and closing discussion.

Each participant started the experiment with handle mode 1 (fixed in a central configuration), then continued with one of the six permutations of the remaining three modes, randomly selected. Since the subjects had to become accustomed to both the instrument and the VR simulator itself during the initial learning session, we decided to always start with the most basic handle mode so as to facilitate the understanding of the VR simulator. In order to check whether this not fully randomized sequence had any influence on the results, the first two subjects repeated the experiment with the first handle mode (five repetitions of the task) at the end of their session, and their performance did not seem to be different from that at beginning of the experiment, suggesting no important cross-learning effect between modes.

\subsection{Statistical analysis}

A total of 120 repetitions ( 6 subjects, 4 handle modes, 5 trials for each mode) were recorded during the experimental session. During 5 trials, the subject let the ring fall and had to redo the entire gesture, increasing task duration and motion economy significantly. These trials were therefore excluded from the performance analysis, although they were taken into account in the ergonomics analysis since the gesture was performed normally. Conversely, recording of the upper limb kinematics did not work during 4 trials and these were not taken into account for the ergonomics analysis, although the performance metrics were recorded properly and included in the statistical analysis.

Valid data were analyzed with JMP 10.0.2 software (SAS Institute Inc.). An ANOVA was performed on the global performance score $P$, time to task completion TTC, motion economy $\mathrm{ME}$, and on the average and maximum values of the RULA-based instantaneous ergonomic score for each trial. Each model contained the following effects: handle mode, subject, and their interaction. The handle mode was defined as a fixed factor, while the subject and the twofactor interaction were defined as random. The linear model was solved using REML (Restricted Maximum Likelihood). The Tukey HSD test of multiple comparisons was used to compare modalities of significant factors.

\section{Results}

Results are depicted on Figs. 5-8. Figures 5 and 7 represent the box-and-whisker plot of the average RULA-based score and the global performance score, respectively. These graphs were derived from the valid data (see above), and indicate the degree of dispersion and skewness in the data. Upper and lower limits of the boxes represent the first and third quartiles, respectively, while the intermediate line is the median. Whiskers depict the lower and higher values, excluding the outliers depicted as dots. These remaining outliers were not excluded from the analysis. Table 1 summarizes the leastsquare means and standard errors modeled by the ANOVA for each ergonomic and performance metric.

The ANOVA performed on the average RULA-based ergonomic score turned out to be significant ( $p=0.0011)$. The Tukey HSD test showed that the first handle mode (standard 
Table 1. Least-squares means and standard errors modeled by ANOVA for each handle mode.

\begin{tabular}{lccccccccc}
\hline & \multicolumn{3}{c}{ Ergonomic scores } & & \multicolumn{4}{c}{ Performance scores } \\
\cline { 2 - 3 } Handle mode & Repetitions & Average RULA & Max. RULA & & Repetitions & $P$ & & TTC & ME \\
\hline Mode 1 & 30 & $3.50(0.171)$ & $4.43(0.216)$ & & 29 & & $34.0(1.76)$ & $29.4(1.48)$ & $3.26(0.25)$ \\
Mode 2 & 29 & $3.03(0.172)$ & $3.88(0.217)$ & & 27 & & $33.2(1.84)$ & $28.2(1.55)$ & $3.55(0.26)$ \\
Mode 3 & 28 & $3.05(0.172)$ & $3.88(0.219)$ & & 30 & $30.2(1.72)$ & $25.8(1.45)$ & $3.15(0.25)$ \\
Mode 4 & 29 & $2.91(0.172)$ & $3.57(0.217)$ & & 29 & & $34.1(1.76)$ & $29.6(1.48)$ & $3.24(0.25)$ \\
\hline
\end{tabular}

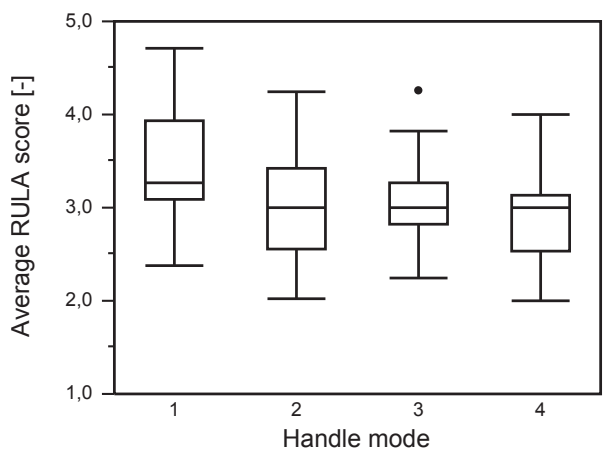

Figure 5. Box-and-whisker plot of the average RULA-based score for each handle mode. Sample size for each box is reported in Table 1. Outliers are represented as dots.

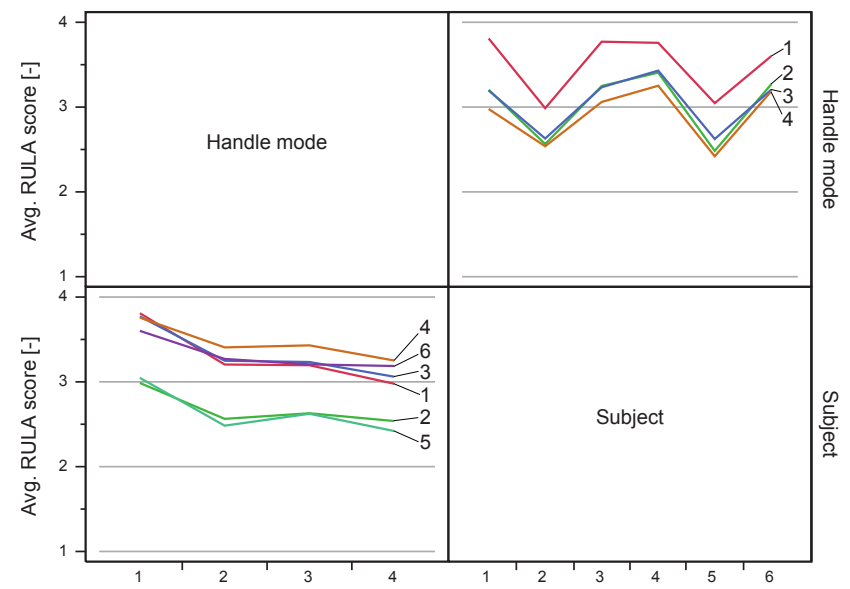

Figure 6. Interaction plot between factors "Handle mode" (fixed) and "Subject" (random) for the average RULA-based score.

fixed handle) was significantly less ergonomic (higher score) than all other modes $(p=0.001$ for mode $4, p=0.0076$ for mode 2 , and $p=0.0114$ for mode 3 ), as can be seen on the upper-right plot of Fig. 6. No difference was found between the other modes $(p>0.63)$. The interaction plot on Fig. 6 suggests that there might be significant differences in terms of average RULA score between two groups of subjects: subjects 2 and 5 have a better (lower) ergonomic score than all the others. However, the plot shows that there is no interaction between factors "handle mode" and "subject": the same

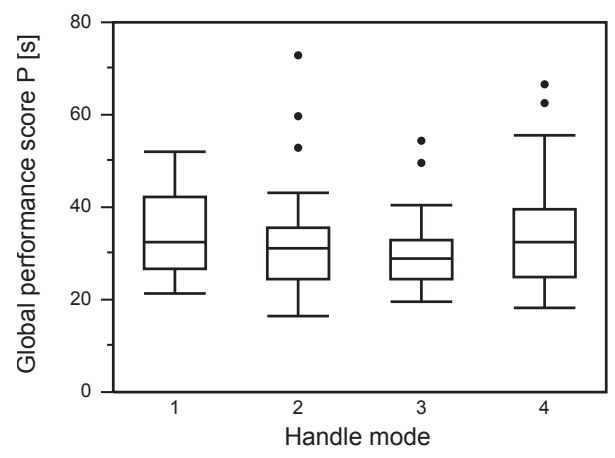

Figure 7. Box-and-whisker plot of the global performance score $P$ for each handle mode. Sample size for each box is reported in Table 1 . Outliers are represented as dots.

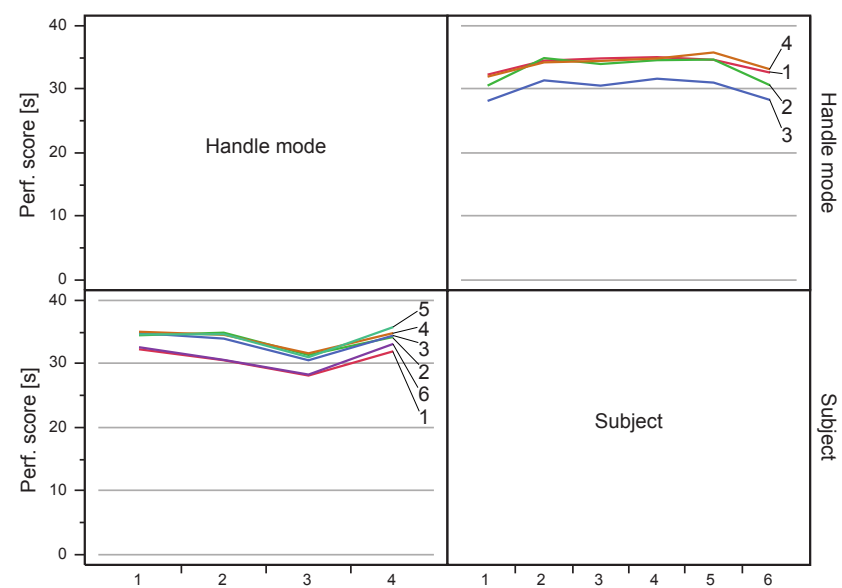

Figure 8. Interaction plot between factors "Handle mode" (fixed) and "Subject" (random) for the global performance score $P$.

handle mode has poorer performance for all subjects. The ANOVA on the maximum ergonomic score is also significant $(p<0.0001)$ and the Tukey HSD results are similar, with a significantly higher value for the first mode ( $p \leq 0.0015)$, no significant difference between the last three modes, and no interaction.

No significant difference in global performance score was found between handle modes $(p=0.42)$. The same result was found for the task duration $(p=0.3)$ and for the motion economy $(p=0.54)$. Differences between subjects are minor, as 
can be seen on Fig. 8, and there is no interaction between factors.

\section{Interpretation}

Figure 6 and the statistical analysis show, as expected, that the ergonomics is improved significantly thanks to the articulated handle. This is in accordance with the previous study (Herman et al., 2011). It confirms the benefits of such a device for laparoscopists, enabling them to operate with a more comfortable and less tiring arm posture than with a handle fixed to the shaft of a conventional or dextrous instrument. In addition, this ergonomic improvement is obtained when the handle is free (mode 2) as in the previous study, and also when it is locked in a convenient configuration (modes 3 and 4 featured by the new design). This confirms our intuition that locking the handle partly or fully does not affect the general arm posture when gestures are concentrated momentarily in a small portion of the intra-abdominal workspace.

This increase in ergonomics is obtained with no decrease in average performance, as depicted in Fig. 8. This was expected for mode 4 where the handle is also fully locked during the task. However, it differs from what we found previously with a free handle (mode 2): in Herman et al. (2011), we reported that a free handle was less efficient than a fixed one. This difference between the two experimental results is due mainly to the fact that rolling the shaft is performed manually using a finger in this experiment, whereas it was motorized and controlled by the joystick previously. This might suggest that the DOFs decoupling proposed in this paper with manual control of the Roll shaft motion is more natural and intuitive than the previous actuated version, although this needs to be confirmed experimentally.

The significant difference found between subjects stems mainly from the fact that the simulator height could only be adjusted between two positions. Depending on his/her own height, each subject chose the most comfortable adjustment. However, arm angles could differ between subjects for the same instrument tip position inside the virtual abdomen, resulting in different average RULA-based scores. In addition, although the same instructions were given repeatedly to the subjects, some tried to maintain the most ergonomic posture possible, while others tried to work as fast as they could and paid less attention to their arm posture.

\section{Conclusions}

Several surgical gestures are difficult to perform using standard laparoscopic instruments. Hand-held robotic instruments with additional end-effector DOFs might be an optimal solution, combining the dexterity enhancement offered by tele-surgery robotic systems and the cost-effectiveness of purely mechanical devices. However, despite these technological improvements, surgeons still have to take uncomfortable, or even painful, postures.

This paper introduces a novel articulated handle that releases constraints between upper limb configuration and instrument tip position and orientation inside the abdominal cavity. An experimental study performed on a custom-made VR simulator tends to demonstrate that the articulated handle helps in restoring an ergonomic arm posture, without reducing gesture performance. A fully-functional instrument prototype is currently being developed for future bench-top and in vivo validation. Ongoing work also includes the implementation of force feedback on the VR simulator, so as to assess its influence on the comparison between instruments.

In addition, although the decrease in ergonomic score offered by the articulated handle is significant, it would be interesting to know to what extent it has an effect on the surgeon's comfort over a period of time longer than the duration of the experiment. A complementary study could therefore be performed using either the VR simulator or the instrument prototype under development. During this study, subjects would repeat gestures with the handle in mode 1 or 3 for at least one hour, and several physiological parameters (e.g. cardiac rythm, EMG in shoulder, arm and forearm muscles) that correlate with physical workload, comfort and ergonomics would be measured.

Finally, although our study focuses only on robotic instruments with intra-abdominal mobility, one can assume that its conclusions could be extended to standard laparoscopic instruments. Since not all surgical gestures require the high dexterity provided by the actuated distal DOFs, standard instruments (e.g. graspers, hooks, scissors) could easily be equipped with such an ergonomic handle and at a low additional cost.

Acknowledgements. The authors would like to warmly thank all their colleagues who took part in the experiments. Authors are also grateful to Catherine Rasse (Institute for Multidisciplinary Research in Quantitative Modelling and Analysis, Université catholique de Louvain) for her valuable advice on the design of this experiment and on the statistical analysis.

Edited by: J. van den Dobbelsteen

Reviewed by: two anonymous referees

\section{References}

Awtar, S., Trutna, T. T., Nielsen, J. M., Abani, R., and Geiger, J.: FlexDex ${ }^{\mathrm{TM}}$ : A minimally invasive surgical tool with enhanced dexterity and intuitive control, J. Med. Devices, 4, 035003, doi:10.1115/1.4002234, 2012.

Barrier, P., Ollagnier, J., and Gautier, G.: Manipulator with decoupled movements and application to instruments for minimally invasive surgery, US Patent Application US2010/0331860A1, 2010. 
Danitz, D. J.: Articulating mechanisms with joint assembly and manual handle for remote manipulation of instruments and tools, US Patent Application US2006/0201130A1, 2006.

Endo, T., Nagasawa, K., Umemura, K., Baba, T., Henmi, H., and Saito, T.: A remarkably easy knot-tying technique for single-incision laparoscopic surgery with the SILS port for gynecologic diseases, J. Minim. Invas. Gyn., 18, 500-502, doi:10.1016/j.jmig.2011.03.014, 2011.

Frede, T., Hammady, A., Klein, J., Teber, D., Inaki, N., Waseda, M., Buess, G., and Rassweiler, J.: The Radius Surgical System: A new device for complex minimally invasive procedures in urology?, Eur. Urol., 51, 1015-1022, 2007.

Freschi, C., Ferrari, V., Melfi, F., Ferrari, M., Mosca, F., and Cuschieri, A.: Technical review of the da Vinci surgical telemanipulator, Int. J. Med. Robot. Comp., 9, 396-406, doi:10.1002/rcs.1468, 2013.

Hackethal, A., Koppan, M., Eskef, K., and Tinneberg, H.-R.: Handheld articulating laparoscopic instruments driven by robotic technology. First clinical experience in gynecological surgery, Gynecol. Surg., 9, 203-206, doi:10.1007/s10397-011-0708-2, 2012.

Hassan Zahraee, A., Paik, J. K., Szewczyk, J., and Morel, G.: Towards the development of a hand-held surgical robot for laparoscopy, IEEE T. Mech., 15, 853-861, doi:10.1109/TMECH.2010.2055577, 2010.

Herman, B., Hassan Zahraee, A., Szewczyk, J., Morel, G., Bourdin, C., Vercher, J.-L., and Gayet, B.: Ergonomic and gesture performance of robotized instruments for laparoscopic surgery, in: Proceedings of the IEEE/RSJ International Conference on Intelligent Robots and Systems (IROS'11), 25-30 September 2011, San Francisco, CA, 1333-1338, doi:10.1109/IROS.2011.6094449, 2011.

Huang, J., Payandeh, S., Doris, P., and Hajshirmohammadi, I.: Fuzzy classification: Towards evaluating performance on a surgical simulator, in: Proceedings of Medicine Meets Virtual Reality (MMVR'05), 26-29 January 2005, Long Beach, CA, 194-200, 2005.

Jinno, M., Matsuhira, N., Sunaoshi, T., Hato, T., Miyagawa, T., Morikawa, Y., Furukawa, T., Ozawa, S., Kitajima, M., and Nakazawa, K.: Development of a master slave combined manipulator for laparoscopic surgery, in: Medical Image Computing and Computer-Assisted Intervention (MICCAI2002), vol. 2488 of Lect. Notes Comput. Sc., edited by: Dohi, T. and Kikinis, R., Springer, Berlin, Heidelberg, 52-59, doi:10.1007/3-540-457860_7, 2002.

Kolwadkar, Y. V., Brown, S. I., Abboud, R. J., and Wang, W.: Comparison of two actuation systems for laparoscopic surgical manipulators using motion analysis, Surg. Endosc., 25, 964-974, doi:10.1007/s00464-010-1300-y, 2011.

Lassooij, J., Tolou, N., Tortora, G., Caccavaro, S., Menciassi, A., and Herder, J. L.: A statically balanced and bi-stable compliant end effector combined with a laparoscopic $2 \mathrm{DoF}$ robotic arm, Mech. Sci., 3, 85-93, doi:10.5194/ms-3-85-2012, 2012.

Lee, W. and Chamorro, A.: Surgical instrument, US Patent US7338513B2, 2008.

MacDonald, E. R., Brownlee, E., and Ahmed, I.: New tools for a new job - Single port laparoscopic surgery equipment, Med. Equip. Insights, 2, 1-7, doi:10.4137/MEI.S9056, 2009.
Marczyk, S., Pribanic, R., and Horton Jr., K. W.: Surgical instrument with articulation and wrist rotation, US Patent Application USUS2013/0012958, 2013.

Matern, U. and Waller, P.: Instruments for minimally invasive surgery: Principles of ergonomic handles, Surg. Endosc., 13, 174-182, doi:10.1007/s004649900934, 1999.

McAtamney, L. and Corlett, E. N.: RULA: A survey method for the investigation of work-related upper limb disorders, Appl. Ergon., 24, 91-99, doi:10.1016/0003-6870(93)90080-S, 1993.

Nguyen, N. T., Ho, H. S., Smith, W. D., Philipps, C., Lewis, C., De Verab, R. M., and Berguer, R.: An ergonomic evaluation of surgeons' axial skeletal and upper extremity movements during laparoscopic and open surgery, Am. J. Surg., 182, 720-724, doi:10.1016/S0002-9610(01)00801-7, 2001.

Okken, L. M., Chmarra, M. K., Hiemstra, E., Jansen, F. W., and Dankelman, J.: Assessment of joystick and wrist control in hand-held articulated laparoscopic prototypes, Surg. Endosc., 26, 1977-1985, doi:10.1007/s00464-011-2138-7, 2012.

Paik, J. K., Morel, G., Vidal, C., and Henri, P.: Surgical Instrument, US Patent Application US2012/0083770A1, 2010.

Person, J., Hodgson, A., and Nagy, A.: Automated highfrequency posture sampling for ergonomic assessment of laparoscopic surgery, Surg. Endosc., 15, 997-1003, doi:10.1007/s004640080155, 2001.

Piccigallo, M., Focacci, F., Tonet, O., Megali, G., Quaglia, C., and Dario, P.: Hand-held robotic instrument for dextrous laparoscopic interventions, Int. J. Med. Robot. Comp., 4, 331-338, doi:10.1002/rcs.214, 2008.

Rettenmaier, M. A., Lopez, K., Graham, C. L., Brown, J. V., John, C. R., Micha, J. P., and Goldstein, B. H.: RealHand high dexterity instruments for the treatment of stage I uterine malignancy, J. Soc. Laparoend., 13, 27-31, 2009.

Röse, A., Wohlleber, C., Kassner, S., Schlaak, H., and Werthschutzky, R.: A novel piezoelectric driven laparoscopic instrument with multiple degree of freedom parallel kinematic structure, in: Proceedings of the IEEE/RSJ International Conference on Intelligent Robots and Systems (IROS 2009), 10-15 October 2009, St. Louis, MO, 2162-2167, doi:10.1109/IROS.2009.5354507, 2009.

Rosenblatt, P. L., Ferzandi, T. R., Hong, S., and Kim, D.: Laparoscopic paravaginal repair with the Autonomy Laparo-Angle device, J. Minim. Invas. Gyn., 14, S151, doi:10.1016/j.jmig.2007.08.659, 2012.

Schwarz, K. M., Schurr, M. O., and Buess, G. F.: Surgical instrument for minimally invasive surgical interventions, US Patent US6913613B2, 2005.

Wang, X., Wang, S., Li, J., Zhang, G., and Wu, Z.: Conceptual design of a novel multi-DoF manual instrument for laparoscopic surgery, Int. J. Med. Robot. Comp., 9, 75-82, doi:10.1002/rcs.1433, 2012.

Yamashita, H., Hata, N., Hashizume, M., and Dohi, T.: Handheld laparoscopic forceps manipulator using multi-slider linkage mechanisms, in: Medical Image Computing and Computer-Assisted Intervention (MICCAI2004), vol. 3217 of Lect. Notes Comput. Sc., edited by: Barillot, C., Haynor, D. R., and Hellier, P., Springer, Berlin, Heidelberg, 121-128, doi:10.1007/978-3-54030136-3_16, 2004. 